\title{
Serotipos y susceptibilidad antimicrobiana de Streptococcus agalactiae
}

\author{
María Angélica Martínez $\mathrm{T}^{\mathbf{1}}$, Alfredo 0 valle $\mathrm{S}^{2}$, \\ Claudia Durán $\mathrm{T}^{1}$, Iván Reid $\mathrm{S}^{3}, \mathrm{G}$ abriela U rriola J ${ }^{4}$, \\ Beatriz Garay $\mathbf{G}^{4}$, Marcela Cifuentes $D^{5}$. \\ Serotypes and antimicrobial \\ susceptibility of Streptococcus \\ agalactiae
}

Background: Streptococcus agalactiae or group B streptococcus, GBS, is the leading cause of neonatal and maternal infections and an opportunistic pathogen in adults with underlying disease. In the last decade, a dramatic increase in the resistance of this microorganism to erythromycin and clindamycin has been observed. Aim: To determine the serotype distribution and antimicrobial susceptibility of isolates of S agalactiae collected from infections and colonization and to assess the genetic mechanisms of macrolide and clindamycin resistance. Material and methods: A total of 100 GBS isolates were collected between 1998 and 2002, in Santiago, Chile. They were isolated from the amniotic fluid from patients with premature rupture of membranes ( 7 isolates), blood from neonatal sepsis (10 isolates), neonate colonizations (2 strains), skin and soft tissue infections (7 isolates), urinary tract infections ( 5 isolates), genital infections (3 isolates), articular fluid (one isolate), and 65 strains were recovered from vaginal colonization55. Results: Serotypes Ia, II and III were the predominant serotypes identified in our study, accounting for 90 (90\%) of the strains. Five isolates belonged to serotypes Ib (5\%) and two (2\%) to serotype V respectively; no strains belonging to serotype IV were found. All strains were susceptible to penicillin G, ampicillin and cefotaxime, and four isolates (4\%) were resistant to both erythromycin (MC $>64 \mu \mathrm{g} /$ $\mathrm{ml}$ ) and clindamycin ( $\mathrm{MC}>64 \mu \mathrm{g} / \mathrm{ml}$ ). The strains had a constitutive macrolide-lincosamide-streptogramin (CMLSB) resistance phenotype and the erm(A) gene was present in the four isolates. Conclusions: Serotypes Ia, II and III were the predominant serotypes in this study. All strains were susceptible to penicillin G, ampicillin and cefotaxime, and four (4\%) strains were resistant to both erythromycin and clindamycin. The cMLSB resistance phenotype, and the erm(A) gene was detected in resistant strains (Rev Méd Chile 2004; 132: 549-55).

(Key Words: Drug resistance, microbial; Serotyping; Streptococcus agalactiae)

Recibido el 16 de septiembre, 2003. Aceptado en versión corregida el 5 de marzo, 2004.

${ }^{1}$ Programa de Microbiología y Micología, Instituto de Ciencias Biomédicas, Facultad de Medicina, Universidad de Chile, Santiago, Chile. ${ }^{2}$ Servicio de Obstetricia y Ginecología, Hospital San Borja Arriarán, Santiago, Chile. ${ }^{3}$ Centro Médico General Science, Santiago, Chile. ${ }^{4}$ Servicio de Bacteriología, Hospital San Juan de Dios, Santiago, Chile. ${ }^{5}$ Servicio de Bacteriología, Hospital San Borja Arriarán, Santiago, Chile.

Correspondencia a: Dra. María Angélica Martínez T. Suecia

1524, Departamento 403, Santiago, Chile. Fax: 7355855.

E-mail: mamartin@med.uchile.cl 
Streptococcus agalactiae (ß-hemolítico grupo B) Oes la etiología más frecuente de meningitis y sepsis neonatal, con una incidencia en Estados Unidos de América de 0,5 casos por 1.000 nacidos vivos, no obstante las medidas de quimioprofilaxis intraparto implementadas en los últimos años en ese país ${ }^{1,2}$. S agalactiae es también una causa importante de morbilidad infecciosa materna y un patógeno oportunista en adultos con enfermedades crónicas predisponentes ${ }^{3,4}$. Aunque la información disponible confirma la susceptibilidad de este microorganismo a penicilina y a otros antibióticos $§$ lactámicos, numerosos estudios han informado, en el último tiempo, la emergencia de resistencia a eritromicina y clindamicina, antibióticos de uso alternativo en la quimioprofilaxis intraparto de pacientes alérgicas a penicilina ${ }^{2,5-13}$.

$\mathrm{S}$ agalactiae se clasifica en 9 serotipos (Ia, Ib, II al VIII) de acuerdo a la estructura de su polisacárido capsular ${ }^{14}$. Se han descrito diferencias geográficas y temporales en la distribución de los serotipos de este microorganismo, como asimismo, porcentajes variables de resistencia a macrólidos entre los diferentes serotipos ${ }^{6-8,13-16}$. A partir de 1990 el serotipo $\mathrm{V}$ ha emergido rápidamente en Norteamérica, Europa y Asia, como causa frecuente de infecciones invasivas en neonatos y adultos y concentrando entre 30 y $68 \%$ de la resistencia a eritromicina reportada para $\mathrm{S}$ agalactiae $e^{6-8,13,14}$

La vigilancia epidemiológica de la distribución de serotipos es importante para el desarrollo y elección de vacunas conjugadas, actualmente en evaluación ${ }^{17,18}$.

Los objetivos de este estudio fueron determinar la susceptibilidad antimicrobiana y distribución de serotipos de cepas de Streptococcus agalactiae aisladas en infecciones y colonizaciones por este microorganismo y caracterizar sus mecanismos de resistencia a eritromicina y clindamicina, en nuestro medio.

\section{Material y MÉTOdos}

Población bacteriana. Se estudiaron 100 cepas de $\mathrm{S}$ agalactiae aisladas en los Hospitales San Borja Arriarán y San Juan de Dios (70 cepas) y Centro Ginecológico General Science (30 cepas) entre 1998 y 2002. Doce cepas fueron aisladas de recién nacidos; 10 de hemocultivos de niños con sepsis neonatal precoz y dos como colonizantes orofaríngeos. Ochenta y ocho cepas fueron aisladas de adultos, de las cuales, 77 fueron obtenidas de embarazadas. Las cepas aisladas de embarazadas fueron obtenidas de líquido amniótico de pacientes con rotura prematura de membranas, 7 cepas; de urocultivos en pacientes con bacteriuria sintomática, 5 cepas; y 65 cepas fueron aisladas como colonizantes vaginales. Las 11 cepas restantes fueron aisladas de infecciones de piel y tejidos blandos, 7 cepas; de infecciones genitales (uretritis, cervicitis y bartolinitis), 3 cepas; y una cepa de líquido articular.

Los microorganismos fueron identificados por sus características bioquímicas: test de CAMP e hidrólisis de hipurato de sodio positivo y ausencia de crecimiento en agar bilis esculina. Las cepas fueron clasificadas en serotipos Ia, Ib, II, III, IV y $\mathrm{V}$, mediante aglutinación con antisueros comerciales (Denka, Seiken, Tokio, Japón) de acuerdo a las instrucciones del fabricante.

Susceptibilidad antimicrobiana. Se determinó la concentración inhibitoria mínima (CIM) mediante la técnica de dilución en agar Mueller-Hinton con 5\% de sangre de cordero de los siguientes antimicrobianos: penicilina $\mathrm{G}$, ampicilina, cefotaxima, clindamicina, enitromicina y tetraciclina. Drogas de potencia conocida fueron donadas gentilmente por el Laboratorio Chile (penicilina G, ampicilina y cefotaxima) y Laboratorio Upjohn (clindamicina). Eritromicina y tetraciclina fueron adquiridas de Sigma (Sigma Co, St Louis, USA). Como inóculo se utilizó $10^{4}$ UFC, dispensado por el inoculador múltiple de Steers. Las placas fueron incubadas por $24 \mathrm{~h}$ a $36^{\circ} \mathrm{C}$ en $3 \% \mathrm{CO}_{2}$. Como cepa control se utilizó la cepa ATCC 49619 de Streptococcus pneumoniae. La interpretación de los resultados se basó en el criterio del National Committee for Clinical Laboratory Standards, NC$\mathrm{CLS}^{19}$. El fenotipo de resistencia a eritromicina fue determinado por la técnica de difusión doble, en agar Mueller-Hinton con 5\% de sangre de cordero, empleando discos de eritromicina $(15 \mu \mathrm{g})$ y clindamicina $(2 \mu \mathrm{g})$ (Oxoid, Inglaterra). Se consideró fenotipo $\mathrm{MLS}_{\mathrm{B}}$ constitutivo $(\mathrm{CMLS}$ ) a la presencia de resistencia a ambos antimicrobianos ${ }^{20}$. Resistencia a eritromicina y clindamicina, con un achatamiento del halo de clindamicina en la zona cercana al disco de eritromicina fue considerada fenotipo $M L S_{B}$ 
inducible $\left(\mathrm{iMLS} \mathrm{S}_{\mathrm{B}}\right.$ ). El fenotipo $\mathrm{M}$ fue determinado por la presencia de resistencia a eritromicina $\mathrm{y}$ sensibilidad a clindamicina.

Determinación de los genes de resistencia. Se determinó la presencia de los genes erm(A), erm(B) y mef(A) en las cepas resistentes a eritromicina, mediante reacción en cadena de la polimerasa (RCP) de acuerdo al procedimiento indicado por Bingen y cols ${ }^{21}$. Para la extracción del ADN, colonias aisladas de las cepas en estudio fueron suspendidas en $100 \mu \mathrm{l}$ de $\mathrm{H}_{2} \mathrm{O}$ destilada. Las suspensiones bacterianas fueron luego sometidas a ebullición por 10 min y rápidamente enfriadas. Para la amplificación del gen erm(A) se emplearon los partidores 5'-GCA TGA CAT AAA CCT TCA-3' y 5'-AGG TTA TAA TGA AAC AGA-3'. Para la amplificación del gen erm(B) se utilizaron los partidores 5'-CGA GTG AAA AAG TAC TCA AAC-3' y 5'-GGC GTG TTT CAT TGC TTG ATG-3' y para la detección de mef(A) se emplearon los partidores 5'-AGT ATC ATT AAT CAC TAG TGC-3' y 5'-TTC TTC TGG TAC TAA AAG TGG-3'21. Los partidores generaron fragmentos de amplificación correspondientes a $206 \mathrm{pb}$ para el gen erm(A), $616 \mathrm{pb}$ para el gen erm(B) y $348 \mathrm{pb}$ del gen mef(A). Las amplificaciones fueron efectuadas en un volumen de $50 \mu$ l conteniendo $2.5 \mathrm{U}$ Taq ADN polimerasa (Promega), $200 \mu \mathrm{M}$ de cada nucleótido trifosfato, $3 \mathrm{mM} \mathrm{MgCl}_{2}$ y $10 \mu \mathrm{M}$ de cada partidor. Los ciclos de amplificación consistieron en $1 \mathrm{~min}$ de denaturación a $95^{\circ} \mathrm{C}$, seguido por 30 ciclos de $45 \mathrm{~s}$ de denaturación a $95^{\circ} \mathrm{C}, 1 \mathrm{~min}$ de anillamiento a $50^{\circ} \mathrm{C}$ y $45 \mathrm{~s}$ de extensión a $72^{\circ} \mathrm{C}$ y un ciclo final de 7 min de extensión a $72^{\circ} \mathrm{C}$. Las cepas controles conteniendo los genes de resistencia erm(B) y mef(A) fueron proporcionadas gentilmente por la Dra. Taid-Kamral y consistieron en Streptococcus pyogenes 02C 1061 y 02C 1064, respectivamente. Como control del proceso de amplificación del gen erm(A), se utilizó una cepa de $\mathrm{S}$ pyogenes aislada en el laboratorio. Como control negativo se utilizó una cepa de S agalactiae sensible a eritromicina. Las amplificaciones fueron efectuadas en un termociclador MJ Research, Modelo MiniCycler ${ }^{\mathrm{MR}}$, USA. Los productos de amplificación fueron analizados por electroforesis en geles de agarosa al 1\%, teñidos con bromuro de etidio y visualización en transiluminador de luz UV.

\section{RESULTADOS}

En la Tabla 1 se aprecia la distribución de serotipos de las 100 cepas de Sagalactiae estudiadas y su origen de aislamiento. Noventa y siete cepas (97\%) pudieron ser asignadas a un serotipo y 3 cepas fueron no tipificables. Los serotipos Ia, II y III fueron los serotipos más frecuentemente aislados, correspondiendo a $90(90 \%)$ de las cepas. El serotipo III fue el serotipo predominante en nuestro estudio e incluyó a 7 de las 10 cepas aisladas en sepsis neonatal y a $24 / 65(36,9 \%)$ de las cepas colonizantes vaginales obtenidas de embarazadas. Cinco cepas pertenecieron al serotipo Ib (5\%) y dos (2\%) al serotipo V. No se detectaron cepas pertenecientes al serotipo IV.

Tabla 1. D istribución de serotipos de S agalactiae según origen de obtención de las cepas

\begin{tabular}{|lcccccccc|}
\hline Origen de las cepas & \multicolumn{7}{c}{ Serotipos (n) } \\
& Ia & Ib & II & III & IV & V & NT & Total \\
\hline Colonizante vaginal & 20 & 3 & 14 & 24 & - & 1 & 3 & 65 \\
Sepsis neonatal & 3 & - & - & 7 & - & - & - & 10 \\
Colonizante neonatal & - & & 1 & 1 & - & - & - & 2 \\
Rotura prematura de membranas & 3 & 1 & 2 & - & - & 1 & - & 7 \\
Infección urinaria & 3 & - & 2 & - & - & - & - & 5 \\
Infección de piel y tejidos blandos & 4 & - & 1 & 2 & - & - & - & 7 \\
Infección genital & - & - & 1 & 2 & - & - & - & 3 \\
Artritis & - & 1 & - & - & - & - & - & 1 \\
Total cepas (n/\%) & $33(33)$ & $5(5)$ & $21(21)$ & $36(36)$ & 0 & $2(2)$ & $3(3) 100(100)$ \\
\hline
\end{tabular}


Tabla 2. Susceptibilidad antimicrobiana in vitro de 100 cepas de $S$ agalactiae

\begin{tabular}{|lrrrr|}
\hline Antimicrobiano & Rango & $\begin{array}{c}\text { CIM } \\
50 \%\end{array}$ & $\begin{array}{c}(\mu \mathrm{g} / \mathrm{ml}) \\
90 \%\end{array}$ & \% Resistencia \\
\hline Penicilina G & $\leq 0,03-0,12$ & $\leq 0,03$ & 0,06 & 0 \\
Ampicilina & $\leq 0,03-0,12$ & $\leq 0,03$ & 0,12 & 0 \\
Cefotaxima & $\leq 0,03-0,25$ & 0,06 & 0,12 & 0 \\
Clindamicina & $0,03->64$ & 0,06 & 0,12 & 4 \\
Eritromicina & $0,03->64$ & 0,06 & 0,12 & 4 \\
Tetraciclina & $0,25->64$ & $>64$ & $>64$ & 75 \\
\hline
\end{tabular}

En la Tabla 2 se muestra la susceptibilidad antimicrobiana de las 100 cepas analizadas. Todas las cepas fueron sensibles a penicilina $\mathrm{G}$, ampicilina y cefotaxima. Cuatro cepas (4\%) presentaron resistencia combinada a eritromicina y clindamicina, con CIM $>64 \mu \mathrm{g} / \mathrm{ml}$ para cada antibiótico. Tres de las cepas resistentes pertenecieron al serotipo III y una al serotipo Ib, como se aprecia en la Tabla 3. Las 4 cepas presentaron un fenotipo MLS constitutivo (cMLS), detectándose el gen ermB en todas ellas. Setenta y cinco cepas (75\%) fueron resistentes a tetraciclina presentando CIM $>64 \mu \mathrm{g} /$ $\mathrm{ml}$ y $4(4 \%)$ cepas presentaron resistencia intermedia (CIM 2-4 $\mu \mathrm{g} / \mathrm{ml}$ ).

\section{DisCUSIÓN}

Los resultados de este estudio demuestran que la mayoría de las cepas de Streptococcus agalactiae aisladas pertenecen a los serotipos Ia, II y III. Con esto se confirman los resultados obtenidos en nuestro trabajo anterior, en que se tipificaron cepas aisladas de infecciones invasivas y de colonizaciones vaginales ${ }^{22}$.

No obstante, se observan algunas diferencias. En la distribución relativa de los serotipos, el III fue el serotipo más frecuentemente aislado en este estudio, aumentando su frecuencia de aislamiento de 22 a 36\%, mientras que el serotipo II, anteriormente el serotipo predominante disminuyó su frecuencia de 38 a 21\%. En cambio la frecuencia de aislamiento del serotipo Ia ha permanecido constante.

Aunque se estudió un número limitado de cepas invasivas neonatales, la mayoría de ellas perteneció al serotipo III, como asimismo consti-
Tabla 3. Serotipos de las cepas de $S$ agalactiae resistentes a eritromicina

\begin{tabular}{|lrcc|}
\hline Serotipo & Cepas aisladas & \multicolumn{2}{c|}{$\begin{array}{c}\text { Cepas resistentes } \\
\text { de cada serotipo } \\
\text { n }\end{array}$} \\
& $\mathrm{n}$ & 0 & 0 \\
\hline Ia & 33 & 1 & 20 \\
Ib & 5 & 0 & 0 \\
II & 21 & 3 & 8,3 \\
III & 36 & 0 & 0 \\
IV & 0 & 0 & 0 \\
V & 2 & 0 & 0 \\
NT & 3 & & \\
\hline
\end{tabular}

NT: No tipificable.

tuyó el serotipo predominante en el tracto genital de las embarazadas. Varios estudios han señalado, en las últimas dos décadas, un aumento en la incidencia del serotipo III en infecciones neonatales y maternas, constituyendo el serotipo más frecuentemente aislado en casos de sepsis neonatal y de meningitis $8,13,14,23$.

El contenido de ácido siálico del polisacárido capsular es el principal factor de virulencia del serotipo $\mathrm{III}^{24-26}$. Cepas aisladas de infecciones invasivas en humanos muestran mayor contenido de ácido siálico capsular y son más resistentes a la fagocitosis que cepas aisladas como colonizantes. El tratamiento con neuraminidasa de este serotipo (no de los serotipos Ia y II), reduce significativamente su virulencia en modelos animales ${ }^{25}$. Por otra parte, la mayor capacidad de adherencia a células endoteliales de las cepas del serotipo III, sugiere que esta caractenística contribuye a causar bacteremias ${ }^{24}$. 
En contraste con lo informado en la literatura extranjera, tanto en este estudio como en el anterior encontramos una baja frecuencia de aislamiento del serotipo $\mathrm{V}^{22}$. Este serotipo es uno de los más frecuentes en la actualidad en infecciones invasivas en adultos con condiciones predisponentes y menos frecuente en infecciones invasivas maternas y neonatales $14,27,28$.

La ausencia de cepas representantes del serotipo IV en nuestro estudio no es sorprendente, ya que este serotipo es detectado con baja frecuencia en infecciones humanas $8,13-16$.

Las cepas de los serotipos VI al VIII no se tipificaron por no estar disponibles comercialmente los antisueros. Como sólo 3 cepas en este estudio fueron no tipificables, es probable que estos serotipos no sean muy frecuentes en Chile, al igual que en otros países ${ }^{15,16}$. En cambio en Japón, en embarazadas colonizadas por S agalactiae, estos serotipos son los más prevalentes. En el futuro, el espectro de serotipos debería aumentar más, ya que algunos estudios actuales señalan hasta $13 \%$ de cepas no tipificables ${ }^{8}$.

En los últimos años se han evaluado vacunas en mujeres no embarazadas, que contienen antígenos capsulares de los serotipos Ia y Ib ó II y III (en forma individual o en combinación), conjugados con toxoide tetánico, con buena capacidad inmunogénica en ensayos de fagocitosis in vitro ${ }^{17,18}$. Recientemente se evaluó una vacuna conjugada conteniendo polisacárido capsular del serotipo III en embarazadas de 30 a 32 semanas de gestación, siendo bien tolerada por las madres y sus hijos ${ }^{29}$. El suero de los neonatos mostró buena capacidad opsonisante y fagocítica al mes y dos meses de vida en ensayos in vitro.

Esto podría significar en un futuro cercano el empleo de vacunas polivalentes en la embarazada, reduciendo la necesidad de efectuar pesquisa y quimioprofilaxis intraparto.

Los resultados de sensibilidad antimicrobiana de nuestro estudio son similares a los encontrados en otros estudios nacionales y extranjeros que confirman la susceptibilidad de S agalactiae a penicilina y otros antibióticos ß lactámicos ${ }^{5-16,30}$. La penicilina es el antibiótico de elección y la ampicilina una alternativa adecuada en la quimioprofilaxis intraparto de la embarazada colonizada con este microorganismo o con factores de riesgo.
Con el uso de estos antimicrobianos se ha demostrado reducción de la incidencia de sepsis neonatal precoz ${ }^{2}$.

Sin embargo, por la emergencia y rápido incremento de la resistencia a eritromicina y clindamicina, antibióticos alternativos en la quimioprofilaxis de pacientes alérgicas a penicilina, es necesario efectuar de rutina el estudio de la susceptibilidad antimicrobiana a estas drogas. Detectamos solamente $4 \%$ de resistencia a eritromicina en nuestro estudio, lo que contrasta con el 7 a $18 \%$ de resistencia publicado en EE.UU. de América, 15 a 21\% reportado en Europa, 33\% en Taiwan y $44 \%$ en Corea $^{5-13}$. La baja prevalencia del serotipo $\mathrm{V}$, que presenta elevada resistencia a macrólidos, podría explicar la escasa resistencia de las cepas chilenas. Belmar y cols, informaron recientemente $1,1 \%$ de resistencia a eritromicina y $3,3 \%$ de resistencia a clindamicina en cepas rectovaginales aisladas de embarazadas ${ }^{30}$. En una publicación anterior encontramos 1,7\% de resistencia a eritromicina y clindamicina ${ }^{31}$. El aumento de la resistencia encontrada en el trabajo actual, probablemente se deba a un incremento real en la resistencia de este microorganismo o diferencias en la población bacteriana estudiada (estudio anterior: mayoritariamente cepas aisladas de infecciones invasivas; presente estudio: cepas preferentemente obtenidas de colonizaciones vaginales).

Se han detectado dos mecanismos de resistencia a macrólidos en cepas de Sagalactiae; modificación del sitio blanco ribosomal por metilación, que es el mecanismo más frecuente y transporte 0 eflujo activo de la droga $a^{5,7,12}$. La metilación del sitio blanco 23S rRNA está mediada por metilasas codificadas por los genes erm(A) y erm(B), otorga resistencia a macrólidos, lincosamidas y estreptogramina $B$ y un fenotipo de resistencia $\mathrm{MLS}_{\mathrm{B}}{ }^{32}$. La expresión de la resistencia puede ser constitutiva o inducible dependiendo de la existencia de secuencias génicas reguladoras río arriba del gen estructural $^{32}$. El mecanismo de eflujo informado en este organismo está mediado por la proteína $\operatorname{Mef}(\mathrm{E})$, codificada por el gen $\operatorname{mef}(\mathrm{E})$. Confiere resistencia a macrólidos, pero no a lincosamidas, lo que se denomina fenotipo $\mathrm{M}^{32}$.

Solamente detectamos 4 cepas resistentes a eritromicina en este estudio, todas ellas presentaron un fenotipo de resistencia $\mathrm{MLS}_{\mathrm{B}}$ constitutivo y 
el gen erm(A). Estudios europeos indican que el principal fenotipo de resistencia en Sagalactiae es el fenotipo $\mathrm{MLS}_{\mathrm{B}}$ inducible, seguido por el fenotipo $\mathrm{MLS}_{\mathrm{B}}$ constitutivo, otorgados por la presencia de los genes erm(A) o erm(B) ${ }^{5,7,12}$. Por otra parte, existe la posibilidad de encontrar otros mecanismos de resistencia para esta bacteria en Chile, ya que Belmar y cols, detectaron un mayor porcentaje de resistencia a clindamicina que a eritromicina

\section{REFERENCIAS}

1. BAKER CJ. Summary of the workshop on perinatal infections due to group B Streptococcus. J Infect Dis 1977; 136: 137-52.

2. CDC. Prevention of perinatal group B streptococcal disease. MMWR 2002/51 (RR11); 1-22.

3. Ovalue A, Gómez R, Martínez Ma, Aspilaga C, Dolz $\mathrm{S}$. Infección vaginal y tratamiento del Streptococcus grupo B en embarazadas con factores universales de riesgo de infección. Resultados neonatales y factores de riesgo de infección neonatal. Rev Chil Obstet Ginecol 2002; 67: 467-75.

4. Farley MM, Harvey RC, Stul T, Smith JM, Schuchat A, WENGER JD ET AL. A population based assessment of invasive disease due to group B Streptococcus in nonpregnant adults. N Engl J Med 1993; 328: 1807-11.

5. De Mouy D, Cavalo J-D, Leclerco R, Fabre R and The AFORCOPI-BIO Network. Antibiotic susceptibility and mechanisms of erythromycin resistance in clinical isolates of Streptococcus agalactiae: French Multiautor Study. Antimicrob Agents Chemother 2001; 45: 2400-2.

6. Fernández M, Hickman ME, BaKer CJ. Antimicrobial susceptibilities of Group B Streptococci isolated between 1992 and 1996 from patients with bacteremia or meningitis. Antimicrob Agents Chemother 1998; 42: 1517-9.

7. Fitoussi F, Loukil $C$, Gros I, Clermont O, Mariani $P$, BONACORSI S ET AL. Mechanisms of macrolide resistance in clinical group B streptococci isolated in France. Antimicrob Agents Chemother 2001; 45: 1889-91. en sus cepas analizadas, lo que sugiere la presencia del fenotipo de resistencia $\mathrm{M}^{30}$.

De acuerdo a los resultados de este estudio, en la infecciones por S agalactiae los serotipos predominantes son Ia, II y III. Todas las cepas son sensibles a penicilina $G$, ampicilina y cefotaxima y algunas resistentes a eritromicina y clindamicina. El mecanismo de resistencia más frecuente es el fenotipo de resistencia $\mathrm{MLS}_{\mathrm{B}}$ constitutivo y el gen erm(A).

8. Ko WC, Lee HC, Wang LR, Lee CT, Lu AJ, Wu JJ. Serotyping and antimicrobial susceptibility of group B Streptococcus over an eight years period in Southern Taiwan. Eur J Clin Microbiol Infect Dis 2001; 20: 334-9.

9. Morales WJ, Dickey SS, Bornick P, Lim DV. Change in antibiotic resistance of group B Streptococcus: Impact on intrapartum management. Am J Obstet Gynecol 1999; 181: 310-4.

10. MuRdoch DR, ReLIER B. Antimicrobial susceptibilities of group B Streptococci isolated from patients with invasive disease: 10 years perspective. Antimicrob Agents Chemother 2001; 45: 3623-4.

11. Pearlman MD, Pierson CL, Faix RG. Frequent resistance in clinical group B streptococci isolates to clindamycin and erythromycin. Obstet Gynecol 1998; 92: 258-61.

12. Portilo A, Lantero M, Olarte I, Ruiz-Larrea F, TORRES C. MLS resistance phenotypes and mechanisms in ß-haemolytic group B, C and G Streptococcus isolated in La Rioja, Spain. J Antimicrob Chemother 2001; 47: 115-6.

13. UH Y, Jang IH, Hwang GY, Yoon KJ, Song W. Emerging erythromycin resistance among Group B Streptococci in Korea. Eur J Clin Microbiol Infect Dis 2001; 20: 52-4.

14. Harrison LH, Eunott JA, Dwyer DM, Libonati JP, FerRieri P, BiLmann L et al. Serotype distribution of invasive group B streptococcal isolated in Maryland: implications for vaccine formulation. J Infect Dis 1998; 177: 998-1002.

15. Lachenauer CS, Kasper DL, Shimada J, Ichiman Y, Ohtsuka H, KaKu M et al. Serotypes VI and VIII predominate among Group B Streptococci isolat- 
ed from pregnant Japanese women. J Infect Dis 1999; 179: 1030-3.

16. Matsubara $K$, Nishiyama $Y$, Katayama $K$, Yamamoto G, Sugiyama M, Murai T et al. Change of antimicrobial susceptibility of group B streptococci over 15 years in Japan. J Antimicrob Chemother 2001; 48: 579-82.

17. Baker CJ, Paoletti LC, Wessels MR, Guttormsen HK, RenCH MA, Hickman ME ET al. Safety and immunogenicity of capsular polysaccharide tetanus toxoid conjugate vaccines for group B streptococcal types Ia and Ib. J Infect Dis 1999; 179: 142-50.

18. Baker CJ, Rench MA, Fernández M, Paoletti LC, KasPer DL, EDWARDS MS. Safety and immunogenicity of a bivalent group B streptococcal conjugate vaccine for serotypes II and III. J Infect Dis 2003; 188: 66-73.

19. National Committee for Cunical Laboratory Standards. Performance Standards for Antimicrobial Susceptibility testing: Twelfth Informational Supplement. M100-S12; 22 (1) 2002. National Committee for Clinical Laboratory Standards, Villanova, Pa.

20. SutclifFe J, Tait-Kamradt A, Wondrack L. Streptococcus pneumoniae and Streptococcus pyogenes resistant to macrolides but sensitive to clindamycin: a common resistance pattern mediated by an efflux system. Antimicrob Agents Chemother 1996; 40: 1817-24.

21. Bingen E, Fitoussi F, Doit C, Cohen R, Tanna A, George $R$ et al. Resistance to macrolides in Streptococcus pyogenes in France in pediatric patients. Antimicrob Agents Chemother 2000; 44: 1453-7.

22. Lämmer C, Schwarz S, Wibawan IWT, Ott E, Bopp B, Martínez-Tagle A. Comparison of streptococci of serological group B isolated from healthy carriers and active disease in Chile. J Med Microbiol 1995; 42: 161-4.

23. Kaliola S, Vuopio-Varkila J, TaKala AK, Eskola J. Neonatal group B streptococcal disease in Finland: a ten years nationwide study. Pediatr Infect Dis J 1999; 18: 806-10.
24. KäLman J, Schown J, Hakansson S, Andersson A, KIHLSTRÖM E. Adherence of group B streptococci to human endothelial cells in vitro. APMIS 1993; 101: 403-8.

25. Shigeoka AO, Rote NS, Santos JI, Hill HR. Assessment of the virulence factors of Group B streptococci: correlation with sialic acid content. J Infect Dis 1983; 147: 857-63.

26. Texeira LA, Figuereido AM, Ferreira BT, Alves VM, Nagao PE, Alviano CS et al. Sialic acid content and surface hydrophobicity of group B streptococci. Epidemiol Infect 1993; 110: 8794.

27. Zaleznik DF, Rench MA, Hiwer S, Krohn MA, Platt $\mathrm{R}$, LEE ML ET AL. Invasive disease due to group B Streptococcus in pregnant women and neonates from diverse population groups. Clin Infect Dis 2000; 30: 276-81.

28. Blumberg HM, Stephens DS, Modansky M, ERwin M, Ешот J, FACKLAM RR et aL. Invasive group B streptococcal disease: the emergence of serotype V. J Infect Dis 1996; 173: 365-73.

29. BAKER CJ, RENCH MA, MCINNES P. Immunization of pregnant women with group B streptococci type III capsular polysaccharide tetanus toxoid conjugate vaccine. Vaccine 2003; 21: 3468-72.

30. Belmar C, Abarzúa F, Becker J, Guzmán AM, García P, Oyarzún E. Estudio de sensibilidad antimicrobiana de 183 cepas de Streptococcus agalactiae aisladas en región vagino-perineal de embarazadas en tercer trimestre. Rev Chile Obstet Ginecol 2002; 67: 106-9.

31. Martínez MA, Ovale A, Giglo MS, Yurac R, Becker J, Campos E et al. Sensibilidad antimicrobiana de Streptococcus agalactiae. Rev Chil Infect 1994; 11: 168-71.

32. Roberts MC, Sutcliffe J, Courvalin P, Jensen LB, Rood J, Seppala H. Nomenclature for macrolide and macrolide-lincosamide-streptogramin $\mathrm{B}$ resistance determinants. Antimicrob Agents Chemother 1999; 43: 2823-30. 